Research Paper

\title{
The Lymphocyte-Monocyte Ratio Predicts Patient Sur- vival and Aggressiveness of Ovarian Cancer
}

\author{
Wan Kyu Eo1, Hye Jung Chang1, Sang Hoon Kwon², Suk Bong Koh³, Young Ok Kim4, Yong Il Ji5, Hong-Bae \\ $\mathrm{Kim}^{6}$, Ji Young Lee ${ }^{7}$, Dong Soo Suh ${ }^{8}$, Ki Hyung Kim ${ }^{8 * \bowtie}$, Ik Jin Chang ${ }^{{ }^{*} \bowtie}$, Heung Yeol Kim ${ }^{9}$, Suk Choo \\ Chang 10 \\ 1. Department of Internal Medicine, College of Medicine, Kyung Hee University, Seoul, Korea; \\ 2. Department of Obstetrics and Gynecology, Keimyung University, School of Medicine, Daegu, Korea; \\ 3. Department of Obstetrics and Gynecology, Catholic University of Daegu, School of Medicine, Daegu, Korea; \\ 4. Department of Pathology, College of Medicine, Kosin University, Busan, Korea; \\ 5. Department of Obstetrics and Gynecology, College of Medicine, Inje University, Busan, Korea; \\ 6. Department of Obstetrics and Gynecology, Kangnam Sacred Heart Hospital, Hallym University Medical Center, Hallym University College of Medicine, Seoul, Korea; \\ 7. Department of Obstetrics and Gynecology, Research Institute of Medical Science, Konkuk University School of Medicine, Seoul, Korea; \\ 8. Department of Obstetrics and Gynecology, Pusan National University School of Medicine; Biomedical Research Institute, Pusan National University Hospital, Busan, \\ Korea; \\ 9. Department of Obstetrics and Gynecology, College of Medicine, Kosin University, Busan, Korea; \\ 10. Department of Plastic and Reconstructive Surgery, College of Medicine, Kyung Hee University, Seoul, Korea. \\ *These authors equally contributed to this work.
}

$\square$ Corresponding author: Ki Hyung Kim, M.D., Ph.D., Department of Obstetrics and Gynecology, Pusan National University School of Medicine; Biomedical Research Institute, Pusan National University Hospital, Busan 602-739, Korea; Phone: +82-51-990-6226; Fax: +82-51-990-3300; e-mail: kihyungkimpnu@gmail.com. Co-correspondence: Ik Jin Chang, M.D., Ph.D., Department of Obstetrics and Gynecology, Kosin University College of Medicine, 34, Amnam-dong, Seo-gu, Busan 602-702, Korea; Phone: +82-51-990-3088; Fax: +82-51-241-5458; E-mail: ijchangku@gmail.com.

(C) Ivyspring International Publisher. Reproduction is permitted for personal, noncommercial use, provided that the article is in whole, unmodified, and properly cited. See http://ivyspring.com/terms for terms and conditions.

Received: 2015.09.18; Accepted: 2015.12.08; Published: 2016.01.29

\begin{abstract}
Objective: To measure the prognostic value of the lymphocyte-monocyte ratio (LMR) in patients with epithelial ovarian cancer (EOC).

Methods: We retrospectively examined the LMR as a prognosticator in a cohort of 234 patients with EOC who underwent surgical resection. Patients were categorized into two different groups based on the LMR (LMR-low and LMR-high) using cut-off values determined by receiver operating characteristic (ROC) curve analysis. The objective of the study was to assess the effect of the LMR on progression-free survival (PFS) and overall survival (OS), and to validate the LMR as an independent predictor of survival.

Results: Using the data collected from the whole cohort, the optimized LMR cut-off value selected on the ROC curve was 2.07 for both PFS and OS. The LMR-low and LMR-high groups included 48 (20.5\%) and 186 patients $(79.5 \%)$, respectively. The 5 -year PFS rates in the LMR-low and LMR-high groups were 40.0 and $62.5 \%(P<0.0001)$, respectively, and the 5-year OS rates in these two groups were 42.2 and $67.2 \%(P<0.0001)$, respectively. On multivariate analysis, we identified age, International Federation of Gynecology and Obstetrics (FIGO) stage, and cancer antigen 125 levels to be the strongest valuable prognostic factors affecting PFS $(P=0.0421, P=0.0012$, and $P=0.0313$, respectively) and age, FIGO stage, and the LMR as the most valuable prognostic factors predicting $O S(P=0.0064, P=0.0029$, and $P=0.0293$, respectively).

Conclusion: The LMR is an independent prognostic factor affecting the survival of patients with EOC.
\end{abstract}

Key words: Monocytes, Lymphocytes, Ovarian neoplasms

\section{Introduction}

Epithelial ovarian cancer (EOC) is one of the important causes of cancer-related deaths in women worldwide [1]. Although curative surgical resection is regarded as the primary treatment modality for ovarian cancer, most ovarian cancers are diagnosed too late [2], and the majority of patients with ad- 
vanced stage disease experience disease recurrence [3]. Considering the poor prognosis, a method for accurately predicting the prognosis of patients with EOC after curative surgical resection is necessary to improve patient survival [4].

In patients with EOC, prognosis is determined based on analysis of cancer-related risk factors. A wide variety of features of high-risk cancer, including size of the tumor, size of the residual tumor [2], stage of the cancer [5], lymph node status [6], histologic type [7], histologic grade [8], malignant ascites [9], and cancer antigen 125 (CA-125) levels [10] are considered essential for predicting cancer-specific survival.

The outcomes of treatments in ovarian cancer patients are determined also by host-related risk factors including performance status, age [11], white blood cell (WBC) [12] and neutrophil counts [13], hemoglobin concentration [12], platelet counts [12], serum albumin levels [14], the neutrophil-lymphocyte ratio (NLR) [15-17], and the platelet-lymphocyte ratio (PLR) $[18,19]$.

The lymphocyte-monocyte ratio (LMR) is calculated as the absolute count of lymphocytes (ALC) divided by the absolute count of monocytes (AMC), and it has been suggested to be associated with survival in patients with malignant lymphomas [20-22] and many solid tumors, including those of head and neck [23-25], breast [26], lung [27-29], gastrointestinal tract [30-37], and genitourinary system [38, 39] cancers. However, to the best of our knowledge, the prognostic value of the LMR in patients with EOC has never been reported. Therefore, the aim of this study was to assess the value of the preoperative LMR for predicting survival in patients with EOC.

\section{Methods}

We retrospectively evaluated 234 patients who underwent primary debulking surgery for EOC at university hospitals between January 2006 and December 2013. Those who had been treated with radiotherapy or neoadjuvant chemotherapy were excluded from the study. Patients with coexisting cancers or prior cancers other than non-melanoma skin cancers within the previous 5 years were also excluded from the study. Moreover, patients were ineligible for inclusion into the study if they had a concurrent autoimmune disease, or had evidence of infection. The retrospective review of these records was approved by the institutional review board, and this study was conducted in accordance with the detailed enforcement regulations of Korea and the principles of the Declaration of Helsinki.

Data including information on patient demographics was collected for analysis. Clinicopatho- logic variables including age, International Federation of Gynecology and Obstetrics (FIGO) stage, histologic type, histologic grade, size of the residual tumor, malignant ascites, and CA-125 levels were obtained retrospectively from patient medical records. Every cytoreduction was principally aimed at maximal tumor resection without visible residual tumor. Standard surgical procedures included midline laparotomy, total extrafascial hysterectomy with bilateral salpingo-oophorectomy, pelvic and paraaortic lymph node dissection, peritonectomy, bowel resection, omentectomy, splenectomy, and partial resection of other affected visceral organs. Ascites was collected after the incision was made for cytologic evaluation. If technically achievable, all visible cancer was resected to achieve optimal tumor debulking (leaving residual tumor $\leq 1 \mathrm{~cm}$ in maximal tumor diameter) [40].

Histologic type classification was reviewed for consistency by a single pathologist. Subtypes included serous, mucinous, endometrioid, clear cell, and mixed cell tumors [17]. Laboratory measurements, including CBCs and biochemical profile analyses, were conducted prior to the surgical resection as part of the routine evaluation. If numerous $\mathrm{CBC}$ s prior to surgery were available, the one that was performed on the nearby date before the surgical resection was selected for analysis. We determined optimized LMR cutoff values for predicting survival outcomes using receiver operating characteristic (ROC) curve analysis; the best LMR cutoff value for both progression-free survival (PFS) and overall survival (OS) was 2.07. Patients were grouped according to the results of ROC curve analysis as follows: LMR-low (LMR $\leq 2.07)$ and LMR-high groups (LMR > 2.07). Differences in cancerand host-related risk factors including age, FIGO stage, histologic type, histologic grade, optimal debulking (OD), and serum CA-125 levels between the LMR-low and LMR-high groups were analyzed. Independent-samples $t$-tests were used to assess continuous variables, whereas independent-samples chi-squared tests were used to assess categorical variables.

Response to primary adjuvant chemotherapy was evaluated according to RECIST criteria or Gynecologic Cancer Intergroup CA-125 criteria [41, 42]. Complete response (CR) was defined as the disappearance of all tumors for at least 4 weeks with normalization of CA-125 levels.

We also evaluated the impact of LMR differences between groups on both PFS and OS. PFS was defined as the time from chemotherapy initiation to disease progression. OS was measured from the date of surgery until death from any cause. Median PFS and OS were estimated by using Kaplan-Meier analysis, and survival curves were compared using log-rank tests. 
The Cox proportional hazards model was used for univariate analysis. The variable that has been analysis in the univariate analysis includes age, histologic type, histologic grade, FIGO stage, OD, malignant ascites, CA-125 levels, serum albumin levels, WBC counts, absolute neutrophil count (ANC), ALC, AMC, hemoglobin concentration, platelet counts, NLR, PLR, an LMR.

To identify the most important prognostic factors for survival, the multivariate Cox proportional hazards model was used; variables with $P$-values < 0.1 were selected for multivariate analysis. All presented $P$-values are two-sided, and statistical significance was declared at $P<0.05$. Data were analyzed using SPSS statistical software, version 18.0 (SPSS Inc., Chicago, IL, USA).

\section{Results}

The baseline characteristics of the patients are displayed in Table 1. Serous adenocarcinoma was the most common subtype (56.4\%), and histologic grade 3 was the most frequent grade $(48.3 \%)$ in our cohort. Endometriosis was additionally present in 20 patients (12.4\%). In total, 82 (35.0\%), 15 (6.4\%), 118 (50.4\%), and $19(8.1 \%)$ patients had stage I, II, III, and IV disease, respectively. OD was performed in 203 (86.8\%) patients. Malignant ascites was observed in 124 patients (53.0\%). Response assessments to primary adjuvant chemotherapy were available for 211 patients, and CR was achieved in 147 (69.7\%) patients. The median serum level of CA-125 was 290 units/mL, and the median serum albumin level was $4.2 \mathrm{~g} / \mathrm{dL}$.

Regarding the stratification of patients according to the LMR, the LMR-low and LMR-high groups included $48(20.5 \%)$ and $186(79.5 \%)$ patients, respectively. To evaluate the relevance of the LMR, we assessed differences in the baseline characteristics of the patients according to the different LMR categories. Significant differences of mean between the LMR-low and LMR-high groups were found in the following continuous variables: age $(P=0.0341)$, serum CA-125 levels $(P<0.0001)$, serum albumin levels $(P<0.0001)$, WBC count $(P<0.0001)$, ANC $(P<0.0001)$, ALC $(P<$ $0.0001)$, AMC $(P<0.0001)$, NLR $(P<0.0001)$ and PLR $(P<0.0001)$. Moreover, significant differences were found in categorical variables including FIGO stage $(P$ $<0.0001)$ and malignant ascites $(P<0.0001)$, but not OD, histologic type, or tumor grade (Table 2).

According to Kaplan-Meier analysis, the 5-year PFS rates in the LMR-low and LMR-high groups were 40.0 and $62.5 \%(P<0.0001)$, respectively, and the 5 -year OS rates in the two groups were 42.2 and
67.2\%, respectively $(P<0.0001)$ (Fig. 1). On Kaplan-Meier analysis, the 5-year PFS rates in the lower ( $\leq 62$ years) and higher ( $>62$ years) age groups were 65.2 and $35.0 \%(P<0.0001)$, respectively, and the 5 -year OS rates in these age groups were 69.6 and $39.9 \%$, respectively $(P<0.0001)$. In addition, the 5 -year PFS rates in patients with stage I/II and III/IV disease were 86.9 and $37.3 \%(P<0.0001)$, respectively, and the 5-year OS rates in these two patient groups were 91.4 and $41.6 \%$, respectively $(P<0.0001)$. Finally, the 5-year PFS rates in the lower $(\leq 110.1$ units $/ \mathrm{mL})$ and higher $(>110.1$ units $/ \mathrm{mL}) \mathrm{CA}-125$ groups were 85.2 and $43.0 \%(P<0.0001)$, respectively, and the 5 -year OS rates in these two groups were 81.0 and $51.6 \%$, respectively $(P=0.0013)$ (Fig. 2$)$.

Table 1. Clinicopathologic characteristics of 234 patients with epithelial ovarian cancer

\begin{tabular}{ll}
\hline Variable & Median (range) \\
\hline Age (years) & $54(14-84)$ \\
Histology, $n(\%)$ & $132(56.4)$ \\
$\quad$ Serous & $35(15.0)$ \\
$\quad$ Mucinous & $35(15.0)$ \\
$\quad$ Clear cell & $21(10.0)$ \\
$\quad$ Endometrioid & $5(2.1)$ \\
$\quad$ Mixed epithelial & $6(2.6)$ \\
$\quad$ Other & \\
Histologic grade, $n(\%)$ & $46(19.7)$ \\
$\quad$ G1 & $65(27.8)$ \\
$\quad$ G2 & $113(48.3)$ \\
$\quad$ G3 & $10(4.3)$ \\
$\quad$ Not available & \\
Endometriosis, $n(\%)$ & $205(87.6)$ \\
$\quad$ Absence & $29(12.4)$ \\
Presence & \\
FIGO Stage, $n(\%)$ & $97(41.5)$ \\
$\quad$ I-II & $137(58.5)$ \\
III-IV & \\
Optimal debulking, $n(\%)$ & $31(13.2)$ \\
$\quad$ No & $203(86.8)$ \\
Yes & \\
Malignant ascites, $n(\%)$ & $110(47.0)$ \\
No & $124(53.0)$ \\
Yes & $290(5-17619)$ \\
CA-125 (Unit/mL) & $4.2(1.8-5.3)$ \\
Albumin $(\mathrm{g} / \mathrm{dL})$ & $6790(1530-18000)$ \\
WBC (per $\mu \mathrm{L})$ & $4661.9(981.2-15480.0)$ \\
ANC (per $\mu \mathrm{L})$ & $1496.2(212.7-5215.9)$ \\
ALC (per $\mu \mathrm{L})$ & $426.0(116.3-2003.4)$ \\
AMC (per $\mu \mathrm{L})$ & $12.4(5.2-15.6)$ \\
Hemoglobin $(\mathrm{g} / \mathrm{dL})$ & $300(104-1144)$ \\
Platelet $\left(\times 10^{3} / \mu \mathrm{L}\right)$ & \\
\hline
\end{tabular}

CA-125, cancer antigen 125; ANC, absolute neutrophil count; ALC, absolute lymphocyte count; AMC, absolute monocyte count 

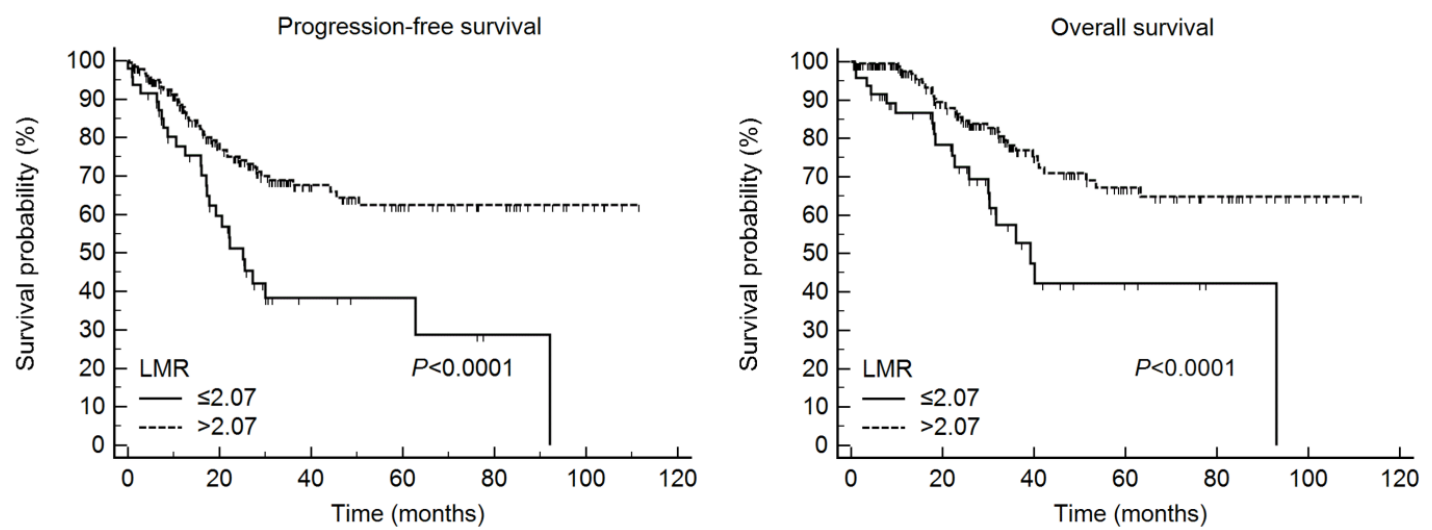

Fig. 1. The LMR predicted progression-free survival and overall survival in 234 patients with epithelial ovarian cancer. LMR, lymphocyte-monocyte ratio
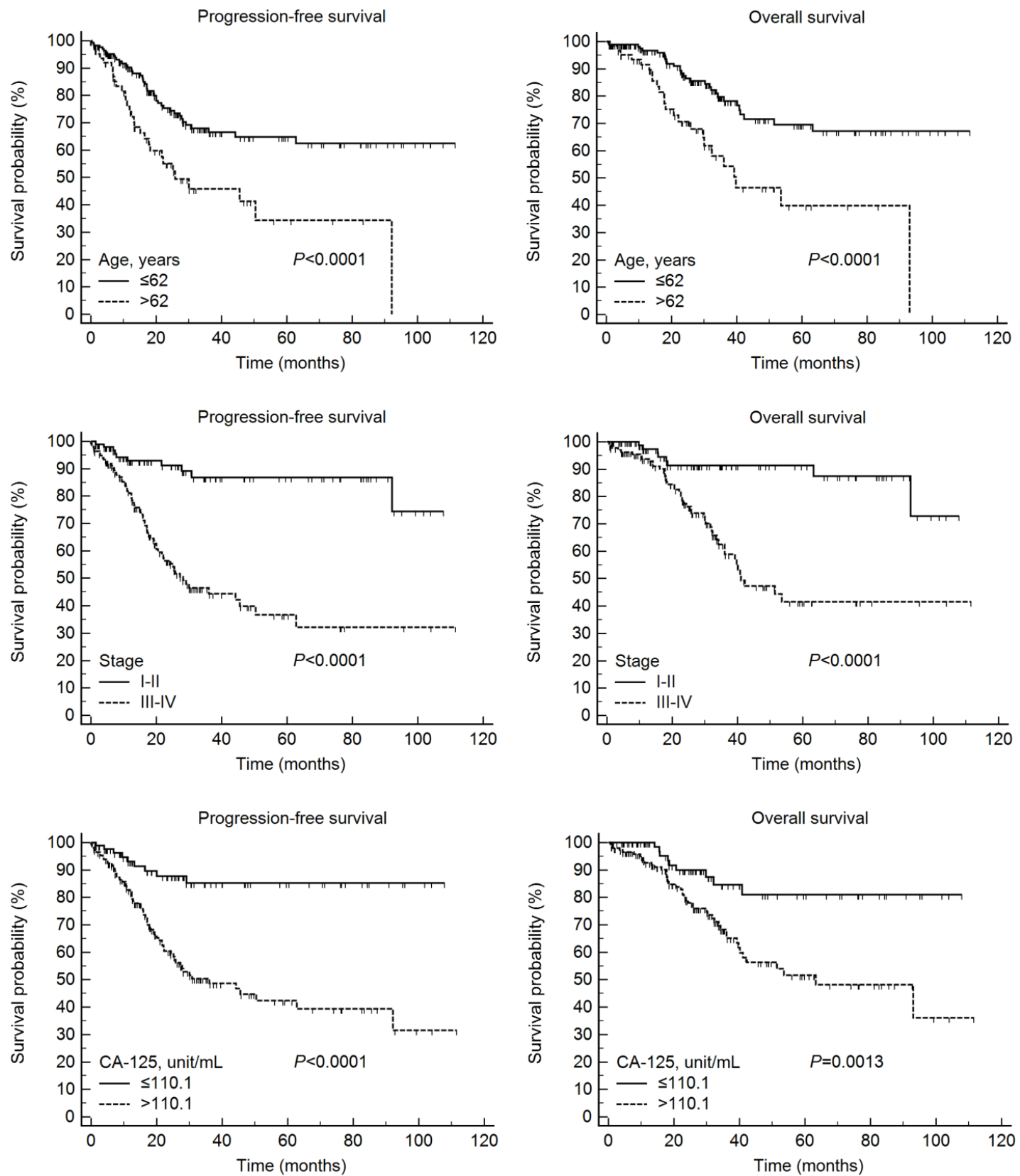

Fig. 2. Progression-free and overall survival according to age, International Federation of Gynecology and Obstetrics stage, and cancer antigen 125 (CA-125) levels in 234 patients with epithelial ovarian cancer. 
Table 2. Clinical and pathologic characteristics according to the lymphocyte-monocyte ratio (LMR) in 234 patients with epithelial ovarian cancer

\begin{tabular}{|c|c|c|c|c|c|c|}
\hline \multirow[t]{2}{*}{ Variable } & & \multicolumn{2}{|r|}{ LMR-low $(\leq 2.07)$} & \multicolumn{2}{|r|}{ LMR-high (> 2.07) } & \multirow[t]{2}{*}{$P$-value } \\
\hline & & $n(\%)$ & Mean \pm SD & $n(\%)$ & Mean \pm SD & \\
\hline Age (years) & & 48 & $58.0 \pm 12.5$ & 186 & $53.7 \pm 12.7$ & 0.0341 \\
\hline \multirow[t]{2}{*}{ Histology } & Serous & 33 & & 99 & & 0.0531 \\
\hline & Non-serous & 15 & & 87 & & \\
\hline \multirow[t]{2}{*}{ Histologic grade } & G1 & 7 & & 39 & & 0.2814 \\
\hline & G2/G3 & 40 & & 138 & & \\
\hline \multirow[t]{2}{*}{ FIGO stage } & I-II & 7 & & 90 & & $<0.0001$ \\
\hline & III-IV & 41 & & 96 & & \\
\hline \multirow[t]{2}{*}{ Optimal debulking } & No & 8 & & 23 & & 0.4332 \\
\hline & Yes & 40 & & 163 & & \\
\hline \multirow[t]{2}{*}{ Malignant ascites } & No & 12 & & 98 & & $<0.0001$ \\
\hline & Yes & 36 & & 88 & & \\
\hline CA-125 (Unit/mL) & & 48 & $1842.7 \pm 3318.6$ & 185 & $681.4 \pm 1486.3$ & $<0.0001$ \\
\hline Albumin (g/dL) & & 48 & $3.3 \pm 0.7$ & 186 & $4.2 \pm 0.5$ & $<0.0001$ \\
\hline WBC (per $\mu \mathrm{L})$ & & 48 & $8876.5 \pm 3551.9$ & 186 & $7044.1 \pm 2321.7$ & $<0.0001$ \\
\hline ANC (per $\mu \mathrm{L})$ & & 48 & $7107.5 \pm 3296.5$ & 186 & $4732.6 \pm 2089.9$ & $<0.0001$ \\
\hline ALC (per $\mu \mathrm{L})$ & & 48 & $992.5 \pm 446.8$ & 186 & $1716.1 \pm 663.6$ & $<0.0001$ \\
\hline $\mathrm{AMC}($ per $\mu \mathrm{L})$ & & 48 & $684.7 \pm 311.8$ & 186 & $401.2 \pm 166.5$ & $<0.0001$ \\
\hline Hemoglobin $(\mathrm{g} / \mathrm{dL})$ & & 48 & $11.9 \pm 1.8$ & 186 & $12.3 \pm 1.5$ & 0.2128 \\
\hline Platelet $\left(\times 10^{3} / \mu \mathrm{L}\right)$ & & 48 & $337.7 \pm 172.9$ & 186 & $308.5 \pm 102.2$ & 0.1335 \\
\hline NLR & & 48 & $8.3 \pm 5.0$ & 186 & $3.2 \pm 2.1$ & $<0.0001$ \\
\hline PLR & & 48 & $406.0 \pm 322.6$ & 186 & $205.4 \pm 103.2$ & $<0.0001$ \\
\hline
\end{tabular}

$P$-values for comparisons of mean differences in continuous variables were obtained using a $t$-test.

$P$-values for comparisons of categorical variables were obtained using the chi-squared test.

CA-125, cancer antigen 125; ANC, absolute neutrophil count; ALC, absolute lymphocyte count; AMC, absolute monocyte count; NLR, neutrophil-lymphocyte ratio; PLR, platelet-lymphocyte ratio

Univariate analysis for PFS identified a significant difference in several variables, including age $(P=$ $0.0005)$, FIGO stage $(P<0.0001)$, OD $(P=0.0268)$, malignant ascites $(P=0.0025)$, CA-125 levels $(P<0.0001)$, serum albumin levels $(P=0.0023)$, ANC $(P=0.0061)$, ALC $(P=0.0027)$, hemoglobin concentration $(P=$ $0.0099)$, platelet counts $(P=0.0338), \mathrm{NLR}(P=0.0005)$, $\operatorname{PLR}(P=0.0042)$, and LMR $(P=0.0012)$. Using the multivariate approach for analysing survival time, we identified age (hazard ratio $[\mathrm{HR}]=1.65,95 \%$ confidence interval $[\mathrm{CI}]=1.02-2.67, P=0.0421)$, FIGO stage $(\mathrm{HR}=3.32,95 \% \mathrm{CI}=1.61-6.85, P=0.0012)$, and CA-125 levels $(\mathrm{HR}=2.28,95 \% \mathrm{CI}=1.08-4.81, P=$ 0.0313 ) as the strongest prognostic factors (Table 3 ).

Univariate analysis for OS identified a significant difference in the same variables found to be significant for PFS: age $(P=0.0002)$, FIGO stage $(P<0.0001)$, OD $(P=0.0018)$, malignant ascites $(P<0.0001)$, CA-125 levels $(P=0.0022)$, serum albumin levels $(P=$ $0.0012)$, ANC $(P=0.0121)$, ALC $(P=0.0290)$, hemoglobin concentration $(P=0.0099)$, platelet counts $(P=$ $0.0285), \operatorname{NLR}(P=0.0103)$, PLR $(P=0.0003)$, and LMR $(P=0.0008)$. Using the multivariate statistical analysis for OS, age $(\mathrm{HR}=2.16,95 \% \mathrm{CI}=1.24-3.76, P=0.0064)$, FIGO stage $(\mathrm{HR}=3.36,95 \% \mathrm{CI}=1.52-7.47, P=0.0029)$, and the LMR $(\mathrm{HR}=0.53,95 \% \mathrm{CI}=0.30-0.94, P=$ 0.0293 ) were identified as significant prognostic factors (Table 4).

\section{Discussion}

EOC is the most lethal gynecologic cancer and one of the major causes of cancer-related death in women worldwide [1]. Although curative surgical resection is the standard treatment for ovarian cancer, most ovarian cancers are diagnosed at an advanced stage because early-stage disease is often asymptomatic $[2,43]$. In addition, the majority of patients with advanced disease experience disease recurrence at some point, resulting in poor survival rates [3]. Considering its poor prognosis, a method for accurately predicting prognosis after curative surgical resection for EOC is required for improving patient survival rates [4].

The association between inflammation and cancer was first described by Virchow in 1863 [44], and emerging evidence has highlighted the importance of chronic inflammation in the malignant transformation, promotion, and metastasis of cancer $[45,46]$. Moreover, inflammatory mediators present in the tumor microenvironment have been found to correlate with chemoresistance in several types of tumors [47]. Pretreatment numbers of peripheral blood cells, including lymphocytes, monocytes, and neutrophils have been suggested to be a significant prognosticator in various types of malignancies. 
Table 3. Relationship of cancer- and host-related characteristics with progression-free survival in 234 patients with epithelial ovarian cancer

\begin{tabular}{|c|c|c|c|c|}
\hline \multirow[b]{2}{*}{ Variable } & \multicolumn{2}{|c|}{ Univariate } & \multicolumn{2}{|c|}{ Multivariate } \\
\hline & HR (95\% CI) & $P$-value & HR $(95 \% \mathrm{CI})$ & $P$-value \\
\hline Age (years) ( $\leq 62$ vs. $>62)$ & $2.32(1.44,3.72)$ & 0.0005 & $1.65(1.02,2.67)$ & 0.0421 \\
\hline \multicolumn{5}{|l|}{ Histology (serous vs. non-serous) } \\
\hline \multicolumn{5}{|l|}{ Histologic grade (G1 vs. G2/G3) } \\
\hline FIGO stage (I-II vs. III-IV) & $5.17(2.63,10.17)$ & $<0.0001$ & $3.32(1.61,6.85)$ & 0.0012 \\
\hline Optimal debulking (yes vs. no) & $1.91(1.08,3.40)$ & 0.0268 & & \\
\hline Malignant ascites (no vs. yes) & $2.27(1.34,3.86)$ & 0.0025 & & \\
\hline CA-125 (Unit/mL ) ( $\leq 110.1$ vs. >110.1) & $4.48(2.23,8.99)$ & $<0.0001$ & $2.28(1.08,4.81)$ & 0.0313 \\
\hline Albumin (g/dL) ( $\leq 3.4$ vs. $>3.4)$ & $0.47(0.29,0.76)$ & 0.0023 & & \\
\hline \multicolumn{5}{|l|}{ WBC $($ per $\mu \mathrm{L})(\leq 6400$ vs. $>6400)$} \\
\hline ANC $($ per $\mu \mathrm{L})(\leq 5053.4$ vs. $>5053.4)$ & $1.92(1.21,3.06)$ & 0.0061 & & \\
\hline ALC (per $\mu \mathrm{L})(\leq 1142.0$ vs. $>1142.0)$ & $0.49(0.31,0.78)$ & 0.0027 & & \\
\hline \multicolumn{5}{|l|}{$\mathrm{AMC}($ per $\mu \mathrm{L})(\leq 327.0$ vs. $>327.0)$} \\
\hline Hemoglobin (g/dL) ( $\leq 12.0$ vs. >12.0) & $0.49(0.29,0.84)$ & 0.0099 & & \\
\hline Platelet $\left(\times 10^{3} / \mu \mathrm{L}\right)(\leq 277$ vs. $>277)$ & $1.75(1.05,2.93)$ & 0.0338 & & \\
\hline $\operatorname{NLR}(\leq 5.03$ vs. $>5.03)$ & $2.34(1.45,3.77)$ & 0.0005 & & \\
\hline PLR $(\leq 246.80$ vs. $>246.80)$ & $1.98(1.24,3.14)$ & 0.0042 & & \\
\hline $\operatorname{LMR}(\leq 2.07$ vs. $>2.07)$ & $0.40(0.23,0.69)$ & 0.0012 & & \\
\hline
\end{tabular}

Hazard ratios were obtained using Cox's proportional hazard model.

$\mathrm{HR}$, hazard ratio; CI, confidence interval; CA-125, cancer antigen 125; ANC, absolute neutrophil count; ALC, absolute lymphocyte count; AMC, absolute monocyte count; NLR, neutrophil-lymphocyte ratio; PLR, platelet-lymphocyte ratio; LMR, lymphocyte-monocyte ratio

Table 4. Relationship of cancer- and host-related characteristics with overall survival in 234 patients with epithelial ovarian cancer

\begin{tabular}{|c|c|c|c|c|}
\hline \multirow[b]{2}{*}{ Variable } & \multicolumn{2}{|c|}{ Univariate } & \multicolumn{2}{|c|}{ Multivariate } \\
\hline & $\mathrm{HR}(95 \% \mathrm{CI})$ & $P$-value & HR $(95 \% \mathrm{CI})$ & $P$-value \\
\hline Age (years) ( $\leq 62$ vs. $>62)$ & $2.74(1.60,4.70)$ & 0.0002 & $2.16(1.24,3.76)$ & 0.0064 \\
\hline \multicolumn{5}{|l|}{ Histology (serous vs. non-serous) } \\
\hline \multicolumn{5}{|l|}{ Histologic grade (G1 vs. G2/G3) } \\
\hline FIGO stage (I-II vs. III-IV) & $4.56(2.13,9.78)$ & $<0.0001$ & $3.36(1.52,7.47)$ & 0.0029 \\
\hline Optimal debulking (yes vs. no) & $2.63(1.43,4.84)$ & 0.0018 & & \\
\hline Malignant ascites (no vs. yes) & $3.28(1.64,6.56)$ & $<0.0001$ & & \\
\hline CA-125 (Unit/mL ) (<110.1 vs. >110.1) & $3.06(1.50,6.25)$ & 0.0022 & & \\
\hline Albumin (g/dL) ( $\leq 3.9$ vs. $>3.9)$ & $0.45(0.23,0.69)$ & 0.0012 & & \\
\hline \multicolumn{5}{|l|}{ WBC $($ per $\mu \mathrm{L})(\leq 6400$ vs. $>6400)$} \\
\hline ANC (per $\mu \mathrm{L})(\leq 4955.0$ vs. $>4955.0)$ & $2.01(1.17,3.44)$ & 0.0121 & & \\
\hline ALC $($ per $\mu \mathrm{L})(\leq 1350.0$ vs. $>1350.0)$ & $0.55(0.32,0.94)$ & 0.0290 & & \\
\hline \multicolumn{5}{|l|}{ AMC $($ per $\mu \mathrm{L})(\leq 327.0$ vs. $>327.0)$} \\
\hline Hemoglobin (g/dL) ( $\leq 12.0$ vs. $>12.0)$ & $0.49(0.29,0.84)$ & 0.0099 & & \\
\hline Platelet $\left(\times 10^{3} / \mu \mathrm{L}\right)(\leq 282$ vs. $>282)$ & $1.98(1.08,3.63)$ & 0.0285 & & \\
\hline $\operatorname{NLR}(\leq 4.28$ vs. $>4.28)$ & $2.01(1.18,3.43)$ & 0.0103 & & \\
\hline PLR ( $\leq 292.50$ vs. $>292.50)$ & $2.70(1.58,4.60)$ & 0.0003 & & \\
\hline LMR ( $\leq 2.07$ vs. $>2.07)$ & $0.40(0.23,0.68)$ & 0.0008 & $0.53(0.30,0.94)$ & 0.0293 \\
\hline
\end{tabular}

Hazard ratios were calculated using Cox's proportional hazard model.

HR, hazard ratio; CI, confidence interval; CA-125, cancer antigen 125; ANC, absolute neutrophil count; ALC, absolute lymphocyte count; AMC, absolute monocyte count; NLR, neutrophil-lymphocyte ratio; PLR, platelet-lymphocyte ratio; LMR, lymphocyte-monocyte ratio

Lymphocytes are considered to play important roles in defenses against cancer cells by inducing cytotoxic cell death and suppressing tumor cell proliferation and migration. It is well accepted that tumor-infiltrating lymphocytes establish a defense barrier against cancer dissemination [45, 48]. Decreased lymphocyte counts in the blood and tumor stroma lead to downregulation of the immune response against tumors [39]. Moreover, decreased lymphocyte counts in the blood has been identified as an independent prognostic factor for OS in various cancers
$[23,49]$. In the present work, ALC was a prognostic factor for both PFS and OS based on univariate analysis but not on multivariate analysis (Tables 3 and 4). When limited to OS for ovarian cancer, our results were compatible with previous findings [15, 17]. Inflammation can trigger the mobilization of monocytes from the bone marrow to the peripheral blood [50]. After recruitment into tumor tissue, monocytes can differentiate into tumor-associated macrophages [51, 52]. Monocytes in the peripheral blood may reflect the formation or presence of tumor-associated macro- 
phages [27]. Moreover, pretreatment numbers of peripheral blood monocytes correlate with poor prognosis in patients with various types of cancers $[28,36]$. In this study, AMC was not a prognostic variable for either PFS or OS (Tables 3 and 4).

In recent years, several prognostic indicators derived from peripheral blood such as NLR and PLR have been widely investigated as useful prognostic markers in cancers. Despite inconsistent results, these markers reportedly have significant diagnostic and prognostic value in a wide variety of cancers. The NLR, the ratio of the ANC to the ALC, has been demonstrated to be a prognostic parameter for various malignancies. Concerning ovarian cancer, the NLR was shown to be associated with worse pathologic features such as advanced tumor stage [16]; an elevated NLR predicted poor PFS [16, 53] or OS [15-17]. In addition, an elevated PLR, a simple ratio between the platelet count and ALC, was associated with a higher rate of advanced disease among patients with ovarian cancer [18], and the impact of an elevated PLR on the survival of women with EOC has been demonstrated $[18,19]$. In the present work, we also found a stage-dependent (stage I/II vs. III/IV) mean difference in both the preoperative NLR (2.99 vs. 5.12, $P<0.0001)$ and PLR (175.03 vs. 297.23, $P<$ 0.0001). Although the prognostic impact of the NLR and PLR on PFS and OS was demonstrated by univariate analysis, the significance of the associations was lost on multivariate analysis (Table 3 and 4).

The LMR, the ratio between the ALC and AMC, has been suggested to be associated with survival in patients with malignant lymphomas [20-22] and many solid tumors, such as head and neck [23-25], breast [26], lung [27-29], esophageal [30,31], gastric $[32,33]$, colorectal $[34,35]$, pancreatic $[36,37]$, bladder [38], and cervical cancers [39]. The cutoff values for the LMR were determined by ROC curve analysis in most studies, and the value ranged from 2.6 to 5.1. A low LMR was associated with poor OS in previous reports [20, 21, 23, 25, 27-31, 34-39], and the LMR can be considered a potential surrogate biomarker in various cancers. Although the mechanisms of the association between lower LMR and poor prognosis have not been fully clarified, the LMR may reflect the balance between the favorable role of lymphocytes and the unfavorable effect of monocytes with respect to cancer progression [25]. The present study demonstrated that the LMR was a surrogate marker for OS, but not PFS on multivariate analysis (Table 3 and 4). The role of the LMR as a prognostic factor for OS, but not relapse- or disease-free survival, has been reported previously for other cancers such as gastric [32] and colorectal cancers [34]. In the present study, a significant difference was observed in the $C R$ rate between the LMR-low and LMR-high groups (48.9\% vs. $75.3 \%, P<0.0001)$. Tumors in patients with an elevated LMR tend to respond better to chemotherapy, and the result of this study was in line with that of previous research [26]. In the present work although the circulating ALC could predict survival outcomes, the LMR was shown to outperform the ALC, which is in line with a previous report [27].

The strength of the current study is that this was the first attempt to evaluate the prognostic value of the LMR in patients with EOC. Moreover, the value of the LMR was evaluated together with previously validated biomarkers, namely the NLR and PLR. In addition, our study was conducted at multiple institutions. Finally, it might be possible to identify patients who are at high risk of experiencing recurrence or death after the current standard treatment by performing simple and low-cost peripheral blood examinations.

This study had some limitations to be addressed, including its retrospective nature and the inclusion of a relatively small number of patients. Another limitation is that the LMR is a non-specific marker of inflammation, and the results may be affected by the presence of other systemic diseases [54]. To apply this convenient, simple, and inexpensive prognostic factor for risk stratification, additional large-scale and standard investigations should be conducted.

In conclusion, this study was the first attempt to assess the prognosis of ovarian cancer patients based on three biomarkers, the NLR, PLR, and LMR. In this study, we identified that an elevated LMR was strongly correlated with longer survival and was an independent prognostic factor for survival in patients with EOC, as determined by multivariate Cox proportional hazards model. Therefore, the LMR may be clinically reliable, and it can be used for accurate prediction of patient prognosis.

\section{Acknowledgements}

This study was supported by Biomedical Research Institute Grant (2013-E3), Pusan National University Hospital.

\section{Competing Interests}

The authors have declared that no competing interest exists.

\section{References}

1. Siegel R, Naishadham D, Jemal A. Cancer statistics, 2013. CA: a cancer journal for clinicians. 2013; 63: 11-30.

2. Polterauer S, Vergote I, Concin N, Braicu I, Chekerov R, Mahner S, et al. Prognostic value of residual tumor size in patients with epithelial ovarian cancer FIGO stages IIA-IV: analysis of the OVCAD data. Int J Gynecol Cancer. 2012; 22: 380-5.

3. Alkema NG, Tomar T, van der Zee AG, Everts M, Meersma GJ, Hollema H, et al. Checkpoint kinase 2 (Chk2) supports sensitivity to platinum-based treatment in high grade serous ovarian cancer. Gynecol Oncol. 2014; 133: 591-8. 
4. Bandera EV, Kushi LH, Rodriguez-Rodriguez L. Nutritional factors in ovarian cancer survival. Nutr Cancer. 2009; 61: 580-6

5. Paik ES, Lee YY, Lee EJ, Choi CH, Kim TJ, Lee JW, et al. Survival analysis of revised 2013 FIGO staging classification of epithelial ovarian cancer and comparison with previous FIGO staging classification. Obstet Gynecol Sci. 2015; 58: 124-34.

6. Bachmann C, Bachmann S, Fehm T, Staebler A, Becker S, Rothmund R, et al. Nodal status--its impact on prognosis in advanced ovarian cancer. Journal of cancer research and clinical oncology. 2012; 138: 261-7.

7. Mizuno M, Kajiyama H, Shibata K, Mizuno K, Kawai M, Nagasaka T, et al. Prognostic value of histological type in stage IV ovarian carcinoma: a retrospective analysis of 223 patients. British journal of cancer. 2015; 112: 1376-83.

8. Makar AP, Baekelandt M, Trope CG, Kristensen GB. The prognostic significance of residual disease, FIGO substage, tumor histology, and grade in patients with FIGO stage III ovarian cancer. Gynecol Oncol. 1995; 56: 175-80.

9. Smolle E, Taucher V, Haybaeck J. Malignant ascites in ovarian cancer and the role of targeted therapeutics. Anticancer research. 2014; 34: 1553-61.

10. Mury D, Woelber L, Jung S, Eulenburg C, Choschzick M, Witzel I, et al. Prognostic and predictive relevance of CA-125 at primary surgery of ovarian cancer. Journal of cancer research and clinical oncology. 2011; 137: 1131-7.

11. Omura GA, Brady MF, Homesley HD, Yordan E, Major FJ, Buchsbaum HJ, et al. Long-term follow-up and prognostic factor analysis in advanced ovarian carcinoma: the Gynecologic Oncology Group experience. Journal of clinical oncology. 1991; 9: 1138-50.

12. Chen Y, Zhang L, Liu WX, Liu XY. Prognostic significance of preoperative anemia, leukocytosis and thrombocytosis in chinese women with epithelial ovarian cancer. Asian Pacific journal of cancer prevention. 2015; 16: 933-9.

13. So KA, Hong JH, Jin HM, Kim JW, Song JY, Lee JK, et al. The prognostic significance of preoperative leukocytosis in epithelial ovarian carcinoma: a retrospective cohort study. Gynecol Oncol. 2014; 132: 551-5.

14. Ataseven B, du Bois A, Reinthaller A Traut A, Heitz F, Aust $\mathrm{S}$, et al Pre-operative serum albumin is associated with post-operative complication rate and overall survival in patients with epithelial ovarian cancer undergoing cytoreductive surgery. Gynecol Oncol. 2015; 138: 560-5.

15. Cho H, Hur HW, Kim SW, Kim SH, Kim JH, Kim YT, et al. Pre-treatment neutrophil to lymphocyte ratio is elevated in epithelial ovarian cancer and predicts survival after treatment. Cancer immunology, immunotherapy. 2009; 58: 15-23.

16. Wang Y, Liu P, Xu Y, Zhang W, Tong L, Guo Z, et al. Preoperative neutrophil-to-lymphocyte ratio predicts response to first-line platinum-based chemotherapy and prognosis in serous ovarian cancer. Cancer Chemother Pharmacol. 2015; 75: 255-62

17. Williams KA, Labidi-Galy SI, Terry KL, Vitonis AF, Welch WR, Goodman A, et al. Prognostic significance and predictors of the neutrophil-to-lymphocyte ratio in ovarian cancer. Gynecol Oncol. 2014; 132. 542-50.

18. Supoken A, Kleebkaow P, Chumworathayi B, Luanratanakorn S, Kietpeerakool C. Elevated preoperative platelet to lymphocyte ratio associated with decreased survival of women with ovarian clear cell carcinoma. Asian Pacific journal of cancer prevention. 2014; 15: 10831-6.

19. Asher V, Lee J, Innamaa A, Bali A. Preoperative platelet lymphocyte ratio as an independent prognostic marker in ovarian cancer. Clinical \& translational oncology. 2011; 13: 499-503.

20. Li ZM, Huang JJ, Xia Y, Sun J, Huang Y, Wang Y, et al. Blood lymphocyte-to-monocyte ratio identifies high-risk patients in diffuse large B-cell lymphoma treated with R-CHOP. PloS one. 2012; 7: e41658.

21. Wang L, Wang H, Xia ZJ, Huang HQ, Jiang WQ, Lin TY, et al. Peripheral blood lymphocyte to monocyte ratio identifies high-risk adult patients with sporadic Burkitt lymphoma. Annals of hematology. 2015; 94: 1645-54.

22. Li YL, Gu KS, Pan YY, Jiao Y, Zhai ZM. Peripheral blood lymphocyte/monocyte ratio at the time of first relapse predicts outcome for patients with relapsed or primary refractory diffuse large B-cell lymphoma. BMC cancer. 2014; 14: 341

23. Lin GN, Peng JW, Liu DY, Xiao JJ, Chen YQ, Chen XQ. Increased lymphocyte to monocyte ratio is associated with better prognosis in patients with newly diagnosed metastatic nasopharyngeal carcinoma receiving chemotherapy. Tumour biology. 2014; 35: 10849-54.

24. Jiang R, Cai XY, Yang ZH, Yan Y, Zou X, Guo L, et al. Elevated peripheral blood lymphocyte-to-monocyte ratio predicts a favorable prognosis in the patients with metastatic nasopharyngeal carcinoma. Chinese journal of cancer. 2015; 34: 237-46.

25. Li J, Jiang R, Liu WS, Liu Q, Xu M, Feng QS, et al. A large cohort study reveals the association of elevated peripheral blood lymphocyte-to-monocyte ratio with favorable prognosis in nasopharyngeal carcinoma. PloS one. 2013; 8: e83069.

26. Ni XJ, Zhang XL, Ou-Yang QW, Qian GW, Wang L, Chen S, et al. An elevated peripheral blood lymphocyte-to-monocyte ratio predicts favorable response and prognosis in locally advanced breast cancer following neoadjuvant chemotherapy. PloS one. 2014; 9: e111886.

27. $\mathrm{Hu} \mathrm{P}$, Shen $\mathrm{H}$, Wang $\mathrm{G}$, Zhang $\mathrm{P}$, Liu $\mathrm{O}$, Du J. Prognostic significance of systemic inflammation-based lymphocyte- monocyte ratio in patients with lung cancer: based on a large cohort study. PloS one. 2014; 9: e108062.

28. Lin GN, Peng JW, Xiao JJ, Liu DY, Xia ZJ. Prognostic impact of circulating monocytes and lymphocyte-to-monocyte ratio on previously untreated metastatic non-small cell lung cancer patients receiving platinum-based doublet. Medical oncology. 2014; 31: 70
29. Go SI, Kim RB, Song HN, Kang MH, Lee US, Choi HJ, et al. Prognostic significance of the lymphocyte-to-monocyte ratio in patients with small cell lung cancer. Medical oncology. 2014; 31: 323.

30. Han LH, Jia YB, Song QX, Wang JB, Wang NN, Cheng YF. Prognostic significance of preoperative lymphocyte-monocyte ratio in patients with resectable esophageal squamous cell carcinoma. Asian Pacific journal of cancer prevention. $2015 ; 16$ : 2245-50.

31. Huang Y, Feng JF. Low preoperative lymphocyte to monocyte ratio predicts poor cancer-specific survival in patients with esophageal squamous cell carcinoma. Onco Targets Ther. 2015; 8: 137-45.

32. Zhou X, Du Y, Xu J, Huang Z, Qiu T, Wang X, et al. The preoperative lymphocyte to monocyte ratio predicts clinical outcomes in patients with stage II/III gastric cancer. Tumour biology. 2014; 35: 11659-66.

33. Deng $\mathrm{Q}, \mathrm{He} B$, Liu $X$, Yue J, Ying $\mathrm{H}$, Pan $\mathrm{Y}$, et al. Prognostic value of pre-operative inflammatory response biomarkers in gastric cancer patients and the construction of a predictive model. J Transl Med. 2015; 13: 66.

34. Ozawa T, Ishihara S, Kawai K, Kazama S, Yamaguchi H, Sunami E, et al. Impact of a lymphocyte to monocyte ratio in stage IV colorectal cancer. J Surg Res. 2015; 199: 386-92.

35. Stotz M, Pichler M, Absenger G, Szkandera J, Arminger F, Schaberl-Moser R, et al. The preoperative lymphocyte to monocyte ratio predicts clinical outcome in patients with stage III colon cancer. British journal of cancer. 2014; 110: $435-40$.

36. Qi Q, Geng Y, Sun M, Wang P, Chen Z. Clinical implications of systemic inflammatory response markers as independent prognostic factors for advanced pancreatic cancer. Pancreatology. 2015; 15: 145-50.

37. Stotz M, Szkandera J, Stojakovic T, Seidel J, Samonigg H, Kornprat P, et al. The lymphocyte to monocyte ratio in peripheral blood represents a novel prognostic marker in patients with pancreatic cancer. Clinical chemistry and laboratory medicine. 2015; 53: 499-506.

38. Zhang GM, Zhu Y, Luo L, Wan FN, Zhu YP, Sun LJ, et al. Preoperative lymphocyte-monocyte and platelet-lymphocyte ratios as predictors of overall survival in patients with bladder cancer undergoing radical cystectomy. Tumour biology. 2015; 36: 8537-43.

39. Chen L, Zhang F, Sheng XG, Zhang SQ. Decreased pretreatment lymphocyte/monocyte ratio is associated with poor prognosis in stage Ib1-IIa cervical cancer patients who undergo radical surgery. Onco Targets Ther. 2015; 8: 1355-62.

40. Braicu EI, Sehouli J, Richter R, Pietzner K, Lichtenegger W, Fotopoulou C. Primary versus secondary cytoreduction for epithelial ovarian cancer: a paired analysis of tumour pattern and surgical outcome. Eur J Cancer. 2012; 48: 687-94.

41. Therasse P, Arbuck SG, Eisenhauer EA, Wanders J, Kaplan RS, Rubinstein L, et al. New guidelines to evaluate the response to treatment in solid tumors. European Organization for Research and Treatment of Cancer, National Cancer Institute of the United States, National Cancer Institute of Canada. J Natl Cancer Inst. 2000; 92: 205-16.

42. Vergote I, Rustin GJ, Eisenhauer EA, Kristensen GB, Pujade-Lauraine E Parmar MK, et al. Re: new guidelines to evaluate the response to treatment in solid tumors [ovarian cancer]. Gynecologic Cancer Intergroup. J Natl Cancer Inst. 2000; 92: 1534-5.

43. Al Rawahi T, Lopes AD, Bristow RE, Bryant A, Elattar A, Chattopadhyay S, et al. Surgical cytoreduction for recurrent epithelial ovarian cancer. Cochrane Database Syst Rev. 2013; 2: CD008765.

44. Balkwill F, Mantovani A. Inflammation and cancer: back to Virchow? Lancet. 2001; 357: 539-45.

45. Coussens LM, Werb Z. Inflammation and cancer. Nature. 2002; 420: 860-7.

46. Grivennikov SI, Greten FR, Karin M. Immunity, inflammation, and cancer. Cell. 2010; 140: 883-99.

47. Wang Y, Niu XL, Qu Y, Wu J, Zhu YQ, Sun WJ, et al. Autocrine production of interleukin-6 confers cisplatin and paclitaxel resistance in ovarian cancer cells. Cancer Lett. 2010; 295: 110-23.

48. Mantovani A, Allavena P, Sica A, Balkwill F. Cancer-related inflammation. Nature. 2008; 454: 436-44

49. Huang JJ, Li YJ, Xia Y, Wang Y, Wei WX, Zhu YJ, et al. Prognostic significance of peripheral monocyte count in patients with extranodal natural killer/T-cell lymphoma. BMC cancer. 2013; 13: 222.

50. Shi C, Pamer EG. Monocyte recruitment during infection and inflammation. Nature reviews Immunology. 2011; 11: 762-74.

51. Pollard JW. Tumour-educated macrophages promote tumour progression and metastasis. Nature reviews Cancer. 2004; 4: 71-8.

52. Hagemann $\mathrm{T}$, Lawrence $\mathrm{T}$. Investigating macrophage and malignant cell interactions in vitro. Methods in molecular biology. 2009; 512: 325-32.

53. Kim HS, Choi HY, Lee M, Suh DH, Kim K, No JH, et al. Systemic Inflammatory Response Markers and CA-125 Levels in Ovarian Clear Cell Carcinoma: A Two Center Cohort Study. Cancer Res Treat. 2015; [Epub ahead of print].

54. Madjid M, Fatemi O. Components of the complete blood count as risk predictors for coronary heart disease: in-depth review and update. Texas Heart Institute journal. 2013; 40: 17-29. 